\title{
Nachhaltigkeit und Innovation: Empirische Befunde und Perspektiven für das Unternehmertum
}

Marcus Wagner, Nicole Weschler, Eva-Maria Lutz

Erschienen in:

Wagner, M., Lutz, E. M., \& Weschler, N. (2014). Nachhaltigkeit und Innovation: Empirische Befunde und Perspektiven für das Unternehmertum. In Pionier-Regionen der Zukunft (pp. 187-208). Springer Gabler, Wiesbaden.

\section{Einleitung}

Das nachhaltige Unternehmertum und nachhaltigkeitsorientierte Innovationen (folgend abgekürzt: Nachhaltigkeitsinnovationen) schaffen durch die Integration von ökologischen, sozialen und ökonomischen Zielen auf der Unternehmensebene, Kooperationen und Lernprozesse den Aufbau spezifischer Kompetenzen letztlich schwer imitierbare Wettbewerbsvorteile. Dabei sind Nachhaltigkeitsinnovationen durch etablierte Unternehmen oder Neugründungen integriert zu betrachten. Nachhaltigkeitsinnovationen und nachhaltiges Unternehmertum schaffen neue Potenziale für regionale Wettbewerbsvorteile und damit letztlich für die Regionalentwicklung insgesamt.

Chapple et al. (2011) argumentieren, dass dabei insbesondere Neugründungen von Netzwerkeffekten in regionalen Clustern profitieren, da diese eher in humankapital-intensiven Industrien innovieren, wohingegen größere etablierte Firmen nachhaltige Innovationen eher in kapitalintensiven Branchen tätigen. Diese Agglomeration von Wissen ist in regionalen Innovationssystemen durch die Präsenz von unterschiedlichen interagierenden Akteuren, Forschungseinrichtungen oder Universitäten gegeben. Empirisch ist laut Chapple et al. (2011) der Einfluss der lokalen Märkte bei der Innovation nachhaltiger Produkte stärker als die Nähe zu Universitäten, wobei die Wachstumschance von Neugründungen wiederum durch Letztere ansteigen. Staatliche Mittel um nachhaltige Innovationen anzustoßen reichen dabei von Regulierungen und Standards über Anreizprogramme und Netzwerkstrategien, wobei die effiziente Kombination von regionalen Gegebenheiten abhängig ist. 
Bisherige Studien zu nachhaltigem Unternehmertum, die die dargestellte Typologie empirisch anwenden zeigen, dass ältere und sehr kleine und sehr große Firmen ohne Umweltmanagementsysteme mit geringerer Wahrscheinlichkeit der Kategorie „Nachhaltiges Unternehmertum“ zugeordnet sind (Wagner 2009a; Wagner/Schaltegger 2010). Darüber hinaus ist nachhaltiges Unternehmertum allerdings keine Frage der Größe. Ebenso können Firmen aus jeder Branche mit gleicher Wahrscheinlichkeit der Kategorie „Nachhaltiges Unternehmertum“ zugeordnet sein. Es gibt eine gewisse negative Assoziation von Unternehmensgröße und der Radikalität der verfolgten Nachhaltigkeitsinnovation(en). Zudem besteht eine Komplementarität zwischen nachhaltigem Unternehmertum kleinerer, jüngerer Firmen und der marktöffnenden Wirkung größerer, etablierter Unternehmen für Nachhaltigkeitsinnovationen. Des Weiteren existieren regionale Schwerpunktsetzungen nachhaltigen Unternehmertums. So werden beispielsweise Wind-, Solarenergie nicht in allen EU-Regionen innoviert.

Grundsätzliche Überlegungen zur Abgrenzung des nachhaltigen Unternehmertums (Walley/Taylor 2002) zeigen, dass sowohl eine enge Orientierung an Nachhaltigkeitsaspekten an sich Auslöser für die Geschäftsidee sein kann, jedoch auch die Bedienung von Nischenmärkten aus opportunistischem Handeln heraus. Einflussfaktoren sind dabei einerseits interne Motivationen, andererseits externe strukturelle Einflüsse wie Regulierungen, ökonomische Anreize oder das soziale Umfeld. Im Folgenden werden zunächst theoretisch die Begrifflichkeiten nachhaltiges Unternehmertum und nachhaltige Innovationen abgegrenzt. Anschließend wird ein Indikatorensystem zur Identifikation und Vergleichbarkeit nachhaltig orientierter Firmen und Innovationen entwickelt, welches zur Validierung exemplarisch auf Fallbeispiele angewendet wird.

\section{Theoriebasierte Identifikation von Kategorien des nachhaltigen Unternehmertums}

In Abhängigkeit der Gewichtung des Nachhaltigkeitsaspekts im Unternehmen lassen sich verschiedene Arten von nachhaltigem Unternehmertum und nachhaltigen Innovationen unterscheiden. Die Kategorisierung basiert auf den beiden Dimensionen Einwirkung von Unternehmen auf spezielle Marktsegmente und dem Grad der Einbindung und Ausführung von ökologischen und sozialen Fragestellungen im Kerngeschäft des Unternehmens. Anhand der folgenden Matrix lassen sich beide Kategorien weiter differenzieren. Die vertikale Achse in Abbildung 1 stellt eine Art Skala dar, anhand derer die Priorität ökologischer und sozialer Fragen als Unternehmensziel gemessen wird. Die Skalenausprägungen variieren zwischen einer geringen (Ökologische und soziale Fragestellungen als eine „Treuhänderpflicht“"), einer mittleren (Nachhaltigkeitsziele ergänzend zum Kerngeschäft) und letztendlich einer hohen Priorität (Nachhaltigkeitsleistungen als ein Kerngeschäft des Unternehmens). Die horizontale Achse in Abbildung 1 bezeichnet die Einwirkung von Unternehmen auf verschiedene Marktsegmente, wie soziale Gruppen, Nischen, Massenmarkt, Markt und Gesellschaft. Eine Bewegung von der linken zur rechten Seite auf der horizontalen Achse hängt von den Innovationen und der Wettbewerbs- und Marktfähigkeit eines Unternehmens ab. Je besser ein Unternehmen wirtschaftet, desto eher kann es weitere Marksegmente erschließen. 


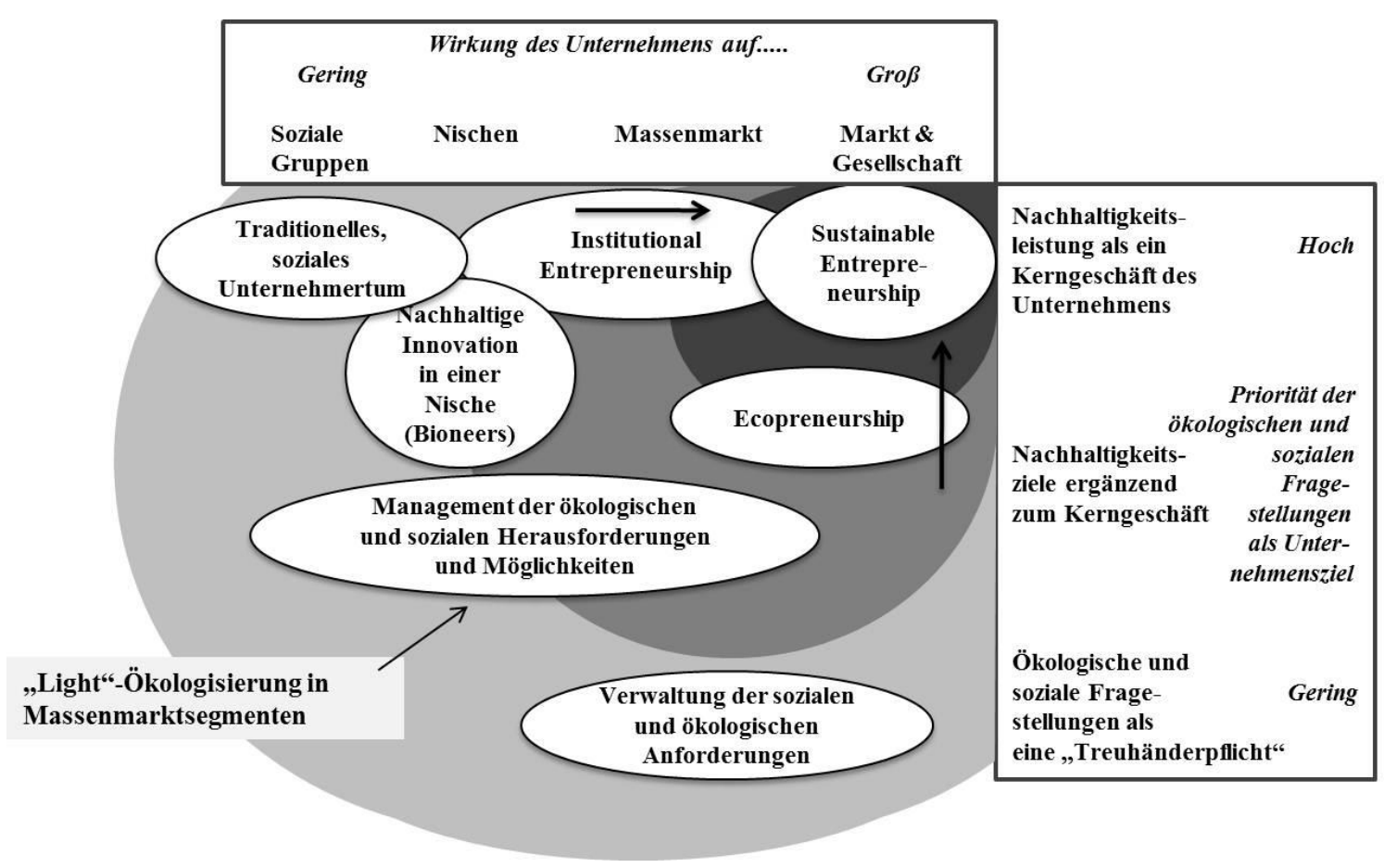

Abbildung 1: Perspektiven und Entwicklung von nachhaltigen Unternehmertum und nachhaltigen Innovationen; Quelle: Eigene, erweiterte Darstellung aufbauend auf Schaltegger/Wagner 2011, Schaltegger/Petersen 2001

„Sustainable Entrepreneurship“ impliziert einerseits die Bereitstellung von nachhaltigen Innovationen und Produkten für den Massenmarkt sowie für die Gesellschaft und andererseits die Verankerung von Nachhaltigkeitsgesichtspunkten im Kerngeschäft des Unternehmens. Werden beide Aspekte erfüllt, kommt dies dem Leitbild von Nachhaltigkeit am nächsten (Schaltegger/Wagner 2011). Somit leisten die „Sustainable Entrepreneurs“ den größten Beitrag zur nachhaltigen Entwicklung. Der Begriff „Ecopreneurship“ (Schaltegger/Petersen 2001) ist eine Kombination der beiden Wörter „ecological“ (deutsch: ökologisch) und „Entrepreneurship“ (deutsch: Unternehmertum). Die Anforderungen eines „Ecopreneurs“ sind eng mit denen eines „Sustainable Entrepreneurs“ verbunden. Der wesentliche Unterschied liegt darin, dass der „Ecopreneur“ stärker ökologische Aspekte im Kerngeschäft des Unternehmens verfolgt und weniger den Fokus auf soziale Zielsetzungen legt. Ebenso orientiert sich das „Institutional Entrepreneurship“ eng am „Sustainable Entrepreneurship“ (Schaltegger/Wagner 2011). Jedoch hat das „Institutional Entrepreneurship“ eine bedeutendere Einwirkung auf den Massenmarkt anstatt auf die Gesellschaft. Eine wichtige Position nehmen auch die „Bioneers“ ein (Schaltegger/Petersen 2001). Die Bezeichnung „Bioneer“ ist ebenfalls eine Wortschöpfung aus den Begriffen „bio“ und „pioneer“ (deutsch: Pionier). Kennzeichen der „Bioneers“ ist deren Sinn für Entwicklung und Forschung. Sie bedienen ein abgegrenztes Marktsegment, also eine Nische mit ökologisch ausgerichteten Produkten. Spezialisierung macht es dem „Bioneer“ möglich, sich erfolgreich am Markt zu positionieren und wettbewerbsfähig zu sein. Gegenüber großen Unternehmen haben „Bioneers“ den Vorteil, dass sie nicht als relevante Konkurrenz angesehen werden. Es ist für große Unternehmen nicht rentabel, sich um die einzelnen Bedürfnisse kleinerer Marktsegmente zu bemühen. Die „Bioneers“ nutzen dementsprechend diese Marktlücke oder auch Nische, um sich gezielt auf eine ausgewählte Kundengruppe zu 
konzentrieren und deren Bedürfnisse zu befriedigen. Die Entwicklung von umweltgerechten Produkten und Umweltinnovationen ist Kern der Philosophie. Infolgedessen werden als Zielgruppe Kunden angesprochen, die ökologische Produkte als wertvoll(er) beurteilen und daher bereit sind, einen entsprechenden Preis dafür zu bezahlen. Als „Traditionelles, soziales Unternehmertum“ bezeichnet man Unternehmen, die die Nachhaltigkeitsleistung als Kernziel des Unternehmens betrachten und vorrangig soziale Gruppen ansprechen. Das „Management der ökologischen und sozialen Herausforderungen und Möglichkeiten“ misst der Nachhaltigkeitsleistung nur mittlere Priorität bei, hat jedoch Einfluss auf unterschiedliche Marktsegmente (Nischen, Massenmarkt, Markt und Gesellschaft). Abschließend misst die Gruppe „Verwaltung der sozialen und ökologischen Anforderungen“ der Integration von ökologischen und sozialen Fragestellungen als Unternehmensziel die geringste Priorität bei. Diese Art von Unternehmertum bezieht sich auf den Massenmarkt sowie Markt und Gesellschaft (Schaltegger/ Wagner 2010, S. 227 ff). Bezüglich der beiden zuletzt genannten Kategorien ist als aktueller Trend festzuhalten, dass eine „Light“-Ökologisierung in Massenmarktsegmenten zu beobachten ist, etwa im Bereich des Lebensmitteleinzelhandels. Anhand dieser Einteilung werden bei der Untersuchung der Praxisfälle in einem späteren Teil des Kapitels die jeweiligen Unternehmen bzw. Projekte den Hauptkategorien Sustainable Entrepreneurs, Bioneer und Sozio-ökologische Manager zugeordnet.

\begin{tabular}{|c|c|c|}
\hline Sozio-ökologischer Manager & Bioneer & Sustainable Entrepreneurs \\
\hline $\begin{array}{l}\text { Ökologisch und soziale Heraus- } \\
\text { forderungen werden eher „verwaltet“ } \\
\text { anstatt aktiv gesteuert }\end{array}$ & Nachhaltigkeit ist zentraler Effekt & $\begin{array}{l}\text { Nachhaltigkeit zentraler Aspekt, } \\
\text { beeinflusst gesamten Markt durch } \\
\text { eigene Nachhaltigkeitsorientierung }\end{array}$ \\
\hline $\begin{array}{l}\text { Fokus auf Umsetzung vorhandener } \\
\text { Regulierungen und Standards }\end{array}$ & Orientiert an Marktnische & $\begin{array}{l}\text { Unternehmenserfolg durch nach- } \\
\text { haltiges Handeln zentraler Bestandteil } \\
\text { der Geschäftstätigkeit }\end{array}$ \\
\hline $\begin{array}{l}\text { Konzentration auf Nischenmarkt, falls } \\
\text { Nachhaltigkeit zentrale Rolle einnimmt }\end{array}$ & & \\
\hline
\end{tabular}

Tabelle 1: Merkmale der Hauptkategorien gemäß Abbildung 1 


\section{Zentrale Merkmale von nachhaltigkeitsorientierten Innovationen}

Obwohl die Bedeutung von Umweltinnovationen immer stärker wahrgenommen wird, gibt es bislang keine eindeutige Abgrenzung des Begriffs. So definieren Reid und Miedzinski (2008) Umweltinnovationen als Entwicklung von neuen und wettbewerbsfähigen Produkten, Prozessen, Systemen, Dienstleistungen und Verfahren, welche menschliche Bedürfnisse befriedigen und eine höhere Lebensqualität bieten, mit einem minimalen Ressourcenverbrauch (Materialien einschließlich Energie und Fläche) pro Outputeinheit über den gesamten Lebenszyklus sowie einer minimalen Freisetzung von toxischen Substanzen. Eine knappere Definition wird vom EIO Eco Innovation Observatory (2010) vorgeschlagen: Eine Umweltinnovation ist jede Innovation, welche den Verbrauch natürlicher Ressourcen und die Freisetzung von schädlichen Stoffen über den gesamten Lebenszyklus hinweg reduziert. Nach dieser Einordnung in den Bereich der Umweltinnovationen können diese, wie Innovationen allgemein, nach dem Innovationsgrad und nach Innovationsarten (Prozess, Produkt, Technologie) unterschieden werden.

Bei der Charakterisierung von Umweltinnovationen stehen vor allem ökologische Aspekte im Vordergrund. Hierzu zählen alle Produkt- und Prozessinnovationsprojekte, die den Umweltfaktor berücksichtigen und sich die Einhaltung von umweltpolitischen Richtlinien zum Ziel gesetzt haben. Umweltinnovationen können in den unterschiedlichsten Bereichen durchgesetzt werden, wie z.B. in Unternehmen, der Politik, privaten Haushalten, Organisationen oder Vereinen. Ausschlaggebend ist die Entwicklung und Einführung neuer Methoden, Prozesse oder Produkte, in deren Fokus die Berücksichtigung ökologischer Aspekte stehen (Rennings 1998, S. 4 f).

Umweltinnovationen unterliegen drei verschiedenen Determinanten (vgl. Abbildung 2). Ein

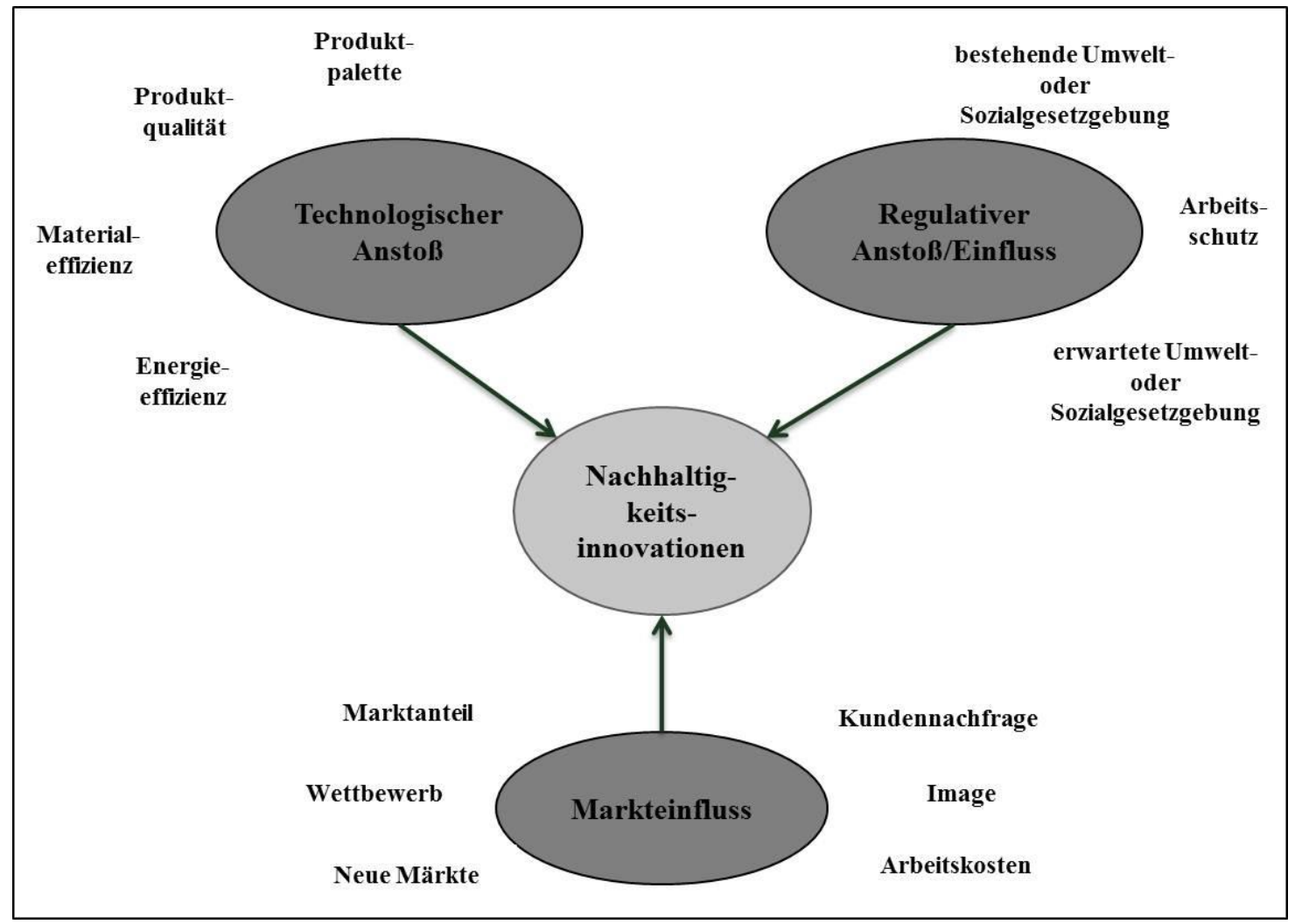

Abbildung 2: Einflussfaktoren von Nachhaltigkeitsinnovationen; Quelle: Eigene, erweiterte Darstellung, aufbauend auf Rennings 2000 
wichtiger Einflussfaktor ist die technologische Determinante, die das Innovationsverhalten im Umweltbereich bestimmt. Die kontinuierliche Steigerung der Energieeffizienz ist beispielsweise ein technologischer Trend. Eine weitere Komponente ist der Einfluss seitens des Marktes. Dabei spielt die Nachfrageseite, vor allem die Präferenzen der Konsumenten, eine enorme Rolle. Tragende Funktion hat der Einflussfaktor regulative Determinante, welche in Form von Regulierungen Innovationen anregen und beschleunigen kann bzw. die Diffusion bereits implementierter Innovationen vorantreibt. Finanzielle Anreize, wie beispielsweise staatliche Subventionen und Steuererleichterungen, tragen zur Förderung von Umweltinnovationen bei bzw. machen diese überhaupt erst möglich. Somit können des Weiteren Umweltinnovationen bezüglich ökologischer Produkt- und Prozessinnovationen unterschieden werden. Ökologische Produktinnovationen unterliegen zum größten Teil der Nachfrage des Marktes (vgl. Markteinfluss). Hierbei handelt es sich um die Entwicklung neuer oder die Verbesserung existierender Produkte. Bei den ökologischen Prozessinnovationen stehen die regulierenden Anforderungen im Vordergrund (vgl. Regulativer Anstoß), welche die Entwicklung eines neuen Produktionsprozesses auslösen (Rennings 2000, S. 326 f). Abbildung 2 stellt hier als Erweiterung der bisherigen Forschung und Literatur neben den drei grundsätzlichen Determinanten von Nachhaltigkeitsinnovationen auch deren Interaktion und hier insbesondere die mögliche und oft real vorhandene moderierende Wirkung von neuen Gesetzesinitiativen und bestehender (z.B. Umwelt)Gesetzgebung und Regulierung auf Markteinfluss (Engl.: market pull) und technologischen Anstoss (Engl.: technology push). Regulativer Anstoss/Einfluss wirkt häufig auch weitestgehend nur durch dieser Interaktions- und Moderationseffekte (vgl. Wagner, 2012).

\section{Definition nachhaltiger Innovationen}

Eine allgemeingültigere Definition von nachhaltigen Innovationen lässt sich unter Berücksichtigung der gesamtwirtschaftlichen Begrifflichkeiten „Externalität“ und „Privater Nutzen“ der Innovation herleiten. Dabei werden unter dem Begriff Nachhaltigkeit sowohl das Streben nach Dauerhaftigkeit und Langfristigkeit der Innovation als auch das Erreichen und die Steigerung der Zielkomplementarität zwischen Ökologie, Ökonomie und Sozialem verstanden. So können nachhaltig orientierte Innovationen zwar nutzensteigernd für die privaten Akteure wirken, gehen aber mit negativen Externalitäten einher. Solange der Private Nutzen die negative Außenwirkung kompensiert, ist der soziale Gesamtnutzen positiv. Diese Situation wird auch dann erreicht, wenn die Innovation den privaten Nutzen zwar senkt, jedoch positive Externalitäten dies kompensieren können. In diesen beiden Fällen liegt der Fall kompensatorisch nachhaltiger Innovationen vor. 


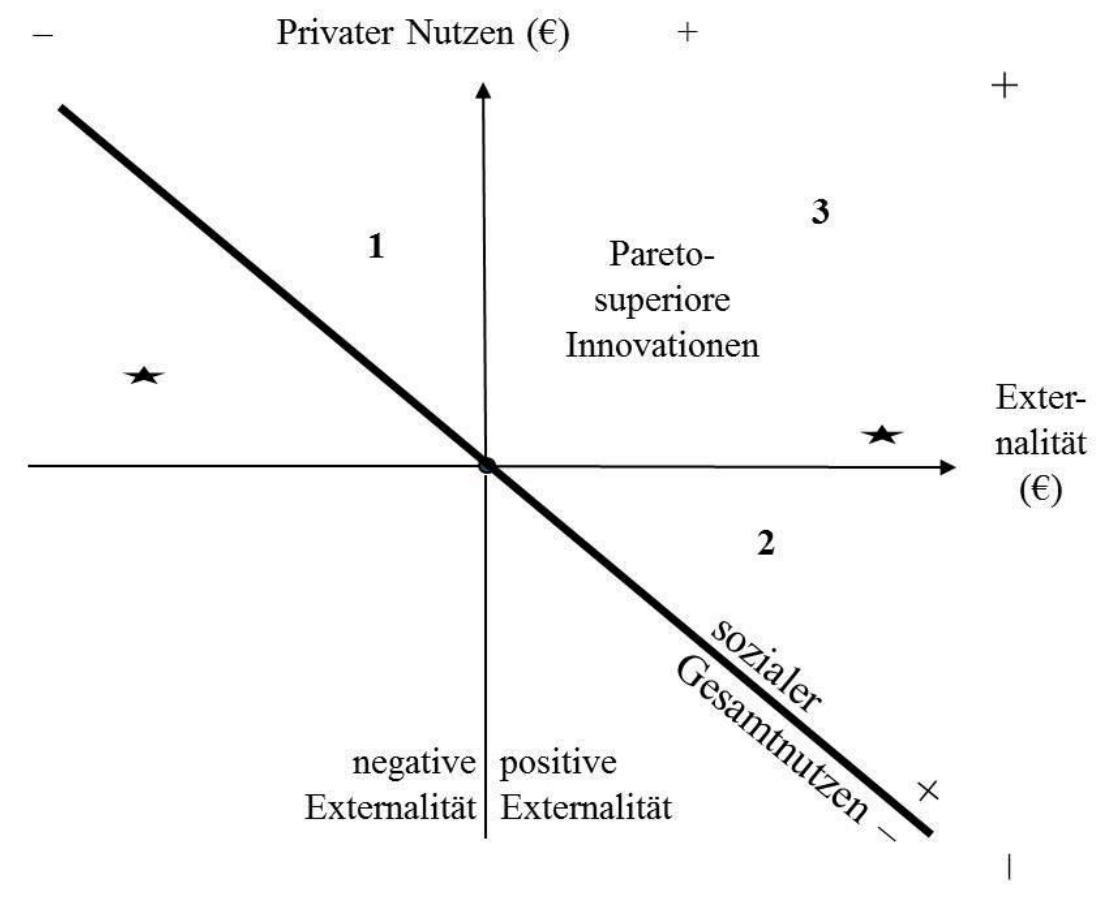

Abbildung 3: Definition von Nachhaltigkeitsinnovationen (Wagner 2009b)

Als pareto-superiore Innovationen können diejenigen bezeichnet werden, welche sowohl den privaten Nutzen steigern als auch gleichzeitig zu positiven Externalitäten führen und somit die Fälle der kompensatorisch nachhaltigen Innovationen strategisch dominieren. Innovationen, welche unterhalb der in der Grafik in Abbildung 3 diagonal verlaufenden Linie unterhalb der Bereiche 1, 2 und 3 liegen, führen entweder zu negativen sozialen Effekten oder haben ein zu geringes Potenzial, um den entgangenen Nutzen vollständig zu kompensieren. Bei positivem privaten Nutzen kann es jedoch zu Crowding-out Effekten kommen. Das heißt es besteht die Gefahr, dass Nachhaltigkeitsinnovationen nicht realisiert werden, wenn alternative Innovationen mit deutlich höherem privaten Nutzen existieren. Sei beispielsweise $\mathrm{S}$ der soziale Nutzen und $\mathrm{P}$ der private Nutzen einer Innovation und mögen zwei Innovationen 1 und 2 existieren, welche zur Auswahl stehen. Dann kann eine Verdrängung auftreten, wenn die Summe aus sozialem und privatem Nutzen aus der Innovation 1 größer ist als die Summe aus sozialem und privatem Nutzen der Innovation 2, wobei der private Nutzen aus Innovation 2 größer sei als aus Innovation 1 ( $\mathrm{S} 1+\mathrm{P} 1>\mathrm{S} 2$ + P2 und P2 > P1.). Dies kann dann der Fall sein, wenn der soziale Nutzen der Innovation 1 wesentlich größer ist als der soziale Nutzen der Innovation 2. Dies würde bedeuten, dass es sich bei Innovation 1 viel eher um eine Nachhaltigkeitsinnovation handelt als bei Innovation 2. Ein Gewinn maximierendes Unternehmen führt jedoch Innovation 2 durch, obwohl Innovation 1 mehr zur sozialen Wohlfahrt beitragen würde. Dies widerspricht dem Argument, dass Innovationen mit positivem privaten Nutzen von Unternehmen auf jeden Fall durchgeführt werden. Ein Beispiel hierfür sind Innovationen zur Steigerung der Energieeffizienz, welche zwar oftmals einen positiven privaten Nutzen haben, im konkreten Einzelfall aber nur dann durchgeführt 
werden, wenn der individuelle private Nutzen höher ist als derjenige der Innovationsalternative mit geringerem sozialen Nutzen.

Zusammenhang von nachhaltigem Unternehmertum und Innovationshöhe von Nachhaltigkeitsinnovationen

Neben der integrierten Betrachtung der verschiedenen Nachhaltigkeitsaspekte ist auch eine Untersuchung des Zusammenhangs zwischen nachhaltigem Unternehmertum und der Innovationshöhe von Nachhaltigkeitsinnovationen sinnvoll, um deren tatsächliches Nachhaltigkeitspotenzial einschätzen zu können. Dabei wird deutlich, dass je tiefgreifender mögliche Veränderungen in das Wissen oder bereits bestehende Systemstrukturen eingreifen und je umfassender die damit verbundenen Beziehungen auf den Märkten oder zwischen Akteuren beeinflusst werden, desto höher ist das jeweilige Nachhaltigkeitspotenzial der Innovation einzustufen.

\section{Nachhaltigkeitspotenzial}

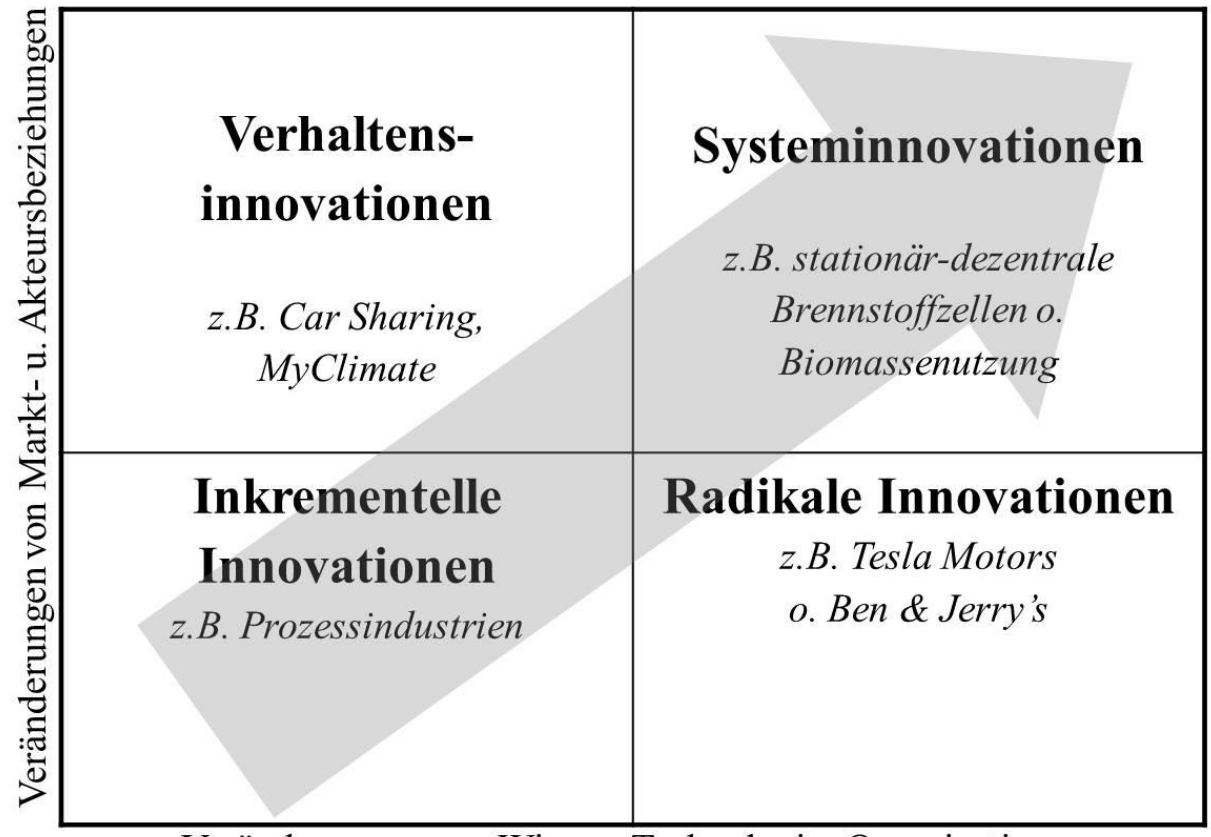

Veränderungen von Wissen, Technologie, Organisation

Abbildung 4: Zusammenhang nachhaltiges Unternehmertum und Innovationshöhe von Nachhaltigkeitsinnovationen; Quelle: in Anlehnung an Konrad/Nill 2001

\section{Relevanz von Vernetzung für nachhaltige Innovation - Cluster-Offensive Bayern}

Die Bedeutung und Stärke regionaler Ansammlungen von Unternehmen einer Branche erhielt durch das von Porter (1990) veröffentlichte Buch „The Competitive Advantage of Nations“ neue Aufmerksamkeit. $\mathrm{Zu}$ unterscheiden sind bei der Wirkungsweise solcher Agglomerationen verschiedene Clustermechanismen. Zum einen können direkte SpilloverEffekte, wie beispielsweise die Weitergabe von spezifischem Wissen, durch die räumliche Nähe von Lieferanten, Kunden oder Forschungseinrichtungen begünstigt werden (Marshall 
1920). Bei der Beurteilung des wirtschaftlichen Erfolgs von Clustern ist allerdings der Konvergenzeffekt innerhalb des Clusters zu berücksichtigen. Mit zunehmender Existenzdauer des Ballungsraums und Größe steigt der Wettbewerb im Cluster z.B. um Produktionsfaktoren und die Zahl der etablierten Unternehmen nimmt zu, was zu höheren Eintrittsbarrieren für Neugründungen führt. Folglich führt dieser Konvergenzprozess $\mathrm{zu}$ einer abnehmenden Wachstumsrate von Unternehmensgründungen mit Alter und Größe des Clusters. Allerdings kommt es in starken Clustern nach Berücksichtigung des Konvergenzeffektes auf Grund von Komplementaritäten dennoch zu einer größeren Anzahl an Unternehmensgründungen, wobei mögliche indirekte Clustereffekte nicht eindeutig bestimmbar sind (Porter 2003; Porter et al. 2010). So ist eine regionale Industriefokussierung (z.B. Kunststoffe, Textil) unter anderem auch von der Art des Wettbewerbs, beispielsweise kosten- oder innovationsbasiert, abhängig. Die Stärke und der Fokus möglicher angrenzender Cluster sind wiederum abhängig von Komplementaritäten bzw. regionalem Wettbewerb insgesamt.

Die Existenz von Clustern und die Präsenz intensiver Netzwerkbeziehungen sind insbesondere für die erfolgreiche Entwicklung und Implementierung von nachhaltigen Innovationen relevant. So sind nachhaltige Innovationen besonders ,vernetzungs- bzw. clusterprädestiniert", da sie auf einen relativ hohen/höheren (geplanten) Innovationsgrad abzielen. Hauschildt (2004) untersucht in diesem Zusammenhang die Dimensionen des Innovationserfolgs und Hauschildt und Salomo (2005) den Zusammenhang mit dem Innovationsgrad. Dabei identifizieren sie direkte und indirekte technische Effekte, ökonomische Effekte (direkt und indirekt) sowie sonstige Effekte (u.a. Umwelt- und Sozialeffekte). Bei nachhaltigen Innovationen spielen intendierte sonstige Effekte als zusätzliche Anforderungen eine entscheidende Rolle.

Um eine große ökologische und soziale Reichweite von Innovationen $\mathrm{zu}$ erreichen, identifizieren Konrad und Nill (2001) als Erfordernisse komplementäre Veränderungen, geeignete Nutzung und breite Nutzungsmöglichkeiten. Dies bedeutet, dass technischorganisatorische Veränderungen allein nicht ausreichen, sondern Veränderungen von Marktund Akteursbeziehungen erforderlich sind. Nachhaltige Innovationen erfordern oftmals ein abgestimmteres Verhalten in den Bereichen Forschung und Entwicklung, Vertrieb und Entsorgung und sind deshalb im Besonderen auf eine Vernetzung angewiesen (Karl \& Müller 2004).

\section{Empirische Analyse von Umweltinnovationen und nachhaltigem Unternehmertum}

Vor dem Hintergrund der oben genannten Erkenntnisse hat die bayerische Staatsregierung im Jahr 2006 die Cluster-Offensive Bayern gestartet, um eine gesteuerte Clusterbildung in bestimmten Zukunftstechnologiefeldern zu fördern. In ausgewählten 21 Branchen werden im Zeitraum 2006 bis 2011 der Aufbau bzw. die Förderung von 19 Clustern angestrebt. Kern der Offensive ist die Vernetzung mit technologieorientierten und -spezifischen Gründer- und Technologiezentren. Die relevantesten Technologiefelder sind dabei Neue Werkstoffe, Chemie, Nanotechnologie, Energietechnik und Umwelttechnologie. Das Konzept konzentriert sich auf die Umsetzung der Cluster-Idee als eine vernetzte Menge von Produzenten, Zulieferern, Dienstleistern und verbundenen Institutionen (IHKs, Universitäten, 
Fachhochschulen) in räumlicher Nähe zueinander, die über Liefer- oder Wettbewerbsbeziehungen interagieren.

Auf den Bereich Umweltwirtschaft fokussiert sich das Umweltcluster Bayern, welches sich schwerpunktmäßig in den Sektoren Abfall- und Kreislaufwirtschaft, Wasser/Abwasser und alternative Energien, insbesondere Energie Biomasse und Abfall engagiert. Das Umweltcluster Bayern ist zudem Mitglied eines europaweiten Netzwerkes aus Umwelttechnologie Clustern (EcoCluP), welches eine Vernetzung von über 3500 Unternehmen und 430 Forschungsinstitutionen aus zehn EU-Ländern darstellt (o.V. 2011).

Ein auf Umwelttechnologie spezialisiertes Gründerzentrum im Rahmen der Cluster-Offensive Bayern ist dabei das UTG Augsburg. Um die zentralen Merkmale von Umweltinnovationen empirisch zu überprüfen, wurden das UTG genauer untersucht. Darüber hinaus wurde in einem zweiten Schritt zur Validierung der entwickelten Kategorien nachhaltigen Unternehmertums ein Evaluationssystem entwickelt und dieses auf drei Fallbeispiele angewendet, um seine Anwendbarkeit zu überprüfen.

\section{Regulierung und Gesetzgebung}

Unter die harten strukturellen Einflussfaktoren, welche Walley und Taylor (2002) als entscheidend für nachhaltiges Unternehmertum identifizieren, fallen auch Regulierungen. Die Wirkung von gesetzlichen Regelungen auf Umweltinnovationen wurde in den letzten Jahrzehnten ausgiebig diskutiert. Befürworter einer strengen Umweltpolitik und -gesetzgebung argumentieren, dass Regulierungen Umweltinnovationen anstoßen können, indem sie die Entwicklung von ressourcenschonenden neue Produkten und Technologien forcieren, welche wiederum die Wettbewerbsfähigkeit der Unternehmen steigern können. Diese Sichtweise wird beispielsweise von Porter und van der Linde (1995) vertreten. Gegner dieses Standpunktes sehen die Wirkung solcher Regulierungen als weitere Belastung für die Unternehmen. Obwohl diese Gesetzgebungen zwar die Öko-Effizienz durch Innovationen steigern können, wird die Wettbewerbssituation beispielsweise durch zusätzliche Kosten negativ beeinflusst. Ein tatsächlicher Einfluss der Regulierungen auf das Innovationsverhalten ist dabei unbestritten, für deren Auswirkungen ist die richtige Kombination und Gestaltung der Gesetzgebungen jedoch entscheidend.

Um ein Regulierungsoptimum zu erreichen, müssen zum einen diese hindernden Effekte vermieden werden und zum anderen gleichzeitig die positiven Impulse der Regulierung für Innovationen maximiert werden. Insgesamt scheinen die abhaltenden Effekte im Fall von radikalen Umweltinnovationen am stärksten und nachteiligsten zu sein. Des Weiteren gibt es Hinweise darauf, dass die Regulierungen insbesondere bei der Diffusion von Umweltinnovationen eine wichtige Rolle spielen. So führten strengere Vorschriften in den USA dazu, dass Volvo zunächst nur dort einen neuen Katalysator einführte. Aufgrund des Inkrafttretens spezifischer Regulierungen in Schweden führten sie diese Innovation dort ebenfalls ein. In die gleiche Richtung zielen weitere Regulierungen wie beispielsweise Subventionen. 


\section{Das UTG Augsburg als Best Practice Beispiel}

Das Umwelttechnologische Gründerzentrum Augsburg GmbH (UTG) wurde 1998 gegründet und ist eines von 400 deutschen Gründerzentren. Der Schwerpunkt des Zentrums, in welchem rund 40 start-up Unternehmen angesiedelt sind, liegt auf ökologischer Gründerförderung aus den Bereichen Energieerzeugung, traditionelle Umwelttechnologien (z.B. Abfall, Abwasser, Lärm, Altlasten) oder Umwelt-Biotechnologie. Dabei ist die Spezialisierung auf umweltbezogene Förderung einzigartig in Europa. Die Messbarkeit von Nachhaltigkeitsbeiträgen der einzelnen Innovationen ist dabei eher schwierig. Die insbesondere im Umweltenergiesektor intensiv diskutierte Anwendung von Messinstrumenten wie Lebenszyklusanalysen sind zu technologiespezifisch, um Vergleichsmaßstäbe daraus entwickeln zu können. Allerdings können diese Messansätze in der individuellen Anwendung von den Unternehmern als Marketinginstrument verwendet werden. Im Durchschnitt verweilten die 100 bisher im UTG geförderten Unternehmen 4,7 Jahre. Dabei variieren die Daten je nach Sektor, so dass z.B. IT-Gründungen zwei bis drei Jahre blieben, wohingegen Umwelttechnologieunternehmen etwa acht Jahre gefördert wurden. Im Allgemeinen beträgt die Dauer bis zur Markteinführung neuer Technologien etwa zehn Jahre (Hehl 2010).

Im Hinblick auf die finanziellen Aspekte profitieren die jungen Firmen im Gründerzentrum von Kostenvorteilen durch relativ niedrige Mietkosten und flexible Vertragsbedingungen. Hinsichtlich der Finanzierung verfolgen die meisten der 1000 Gründerfirmen, die derzeit in bayerischen Gründerzentren angesiedelt sind, eine langfristige Strategie. Die insgesamt schwierige Finanzierungssituation verlangsamt den Prozess der Markteinführung (Hehl 2010). 
Der zweite Schritt der empirischen Analyse beinhaltet aufbauend auf der ersten Stufe die Entwicklung und Validierung eines Indikatorensystems für nachhaltige Innovationen und nachhaltiges Unternehmertum zur Bewertung von und Einordnung in Kategorien des nachhaltigen Unternehmertums bzw. nachhaltiger Innovationen. Da kein allgemeingültiges Standardindikatorensystem existiert, wurde für die Beurteilung ein projektbasiertes Indikatorensystem entwickelt. Die Intention des Indikatorensystems besteht in der Bestimmung von einzelnen Projekten, die als besonders innovativ, effizient und erfolgreich bewertet werden und denen Modell- und Vorbildcharakter zugeschrieben werden kann. Hierzu wird das Indikatorensystem zuerst in sechs unterschiedliche Kategorien unterteilt. Die Kategorisierung ist folgendermaßen aufgebaut: Kategorie eins bis fünf umfassen die drei Dimensionen der Nachhaltigkeit (Ökologie (Punkt 1 und 2), Ökonomie (Punkt 3) und Soziales (Punkt 4 und 5)). Der Unterpunkt sechs, „Innovationen“, fokussiert die wichtigsten Innovationsaspekte wie Neuartigkeit, Modellcharakter und auch die subjektive Bewertung der Bedeutsamkeit dieser für die Zukunft des Prozesses oder des Produkts. Unter Berücksichtigung dieser Kriterien der oben genannten Kategorien wird eine weitere Differenzierung in neunzehn unterschiedliche Indikatoren vorgenommen. Diese Indikatoren werden anschließend unter anderem auf Basis der ersten Stufe der Analyse hinsichtlich ihrer Relevanz für den Aspekt der Nachhaltigkeit gewichtet.

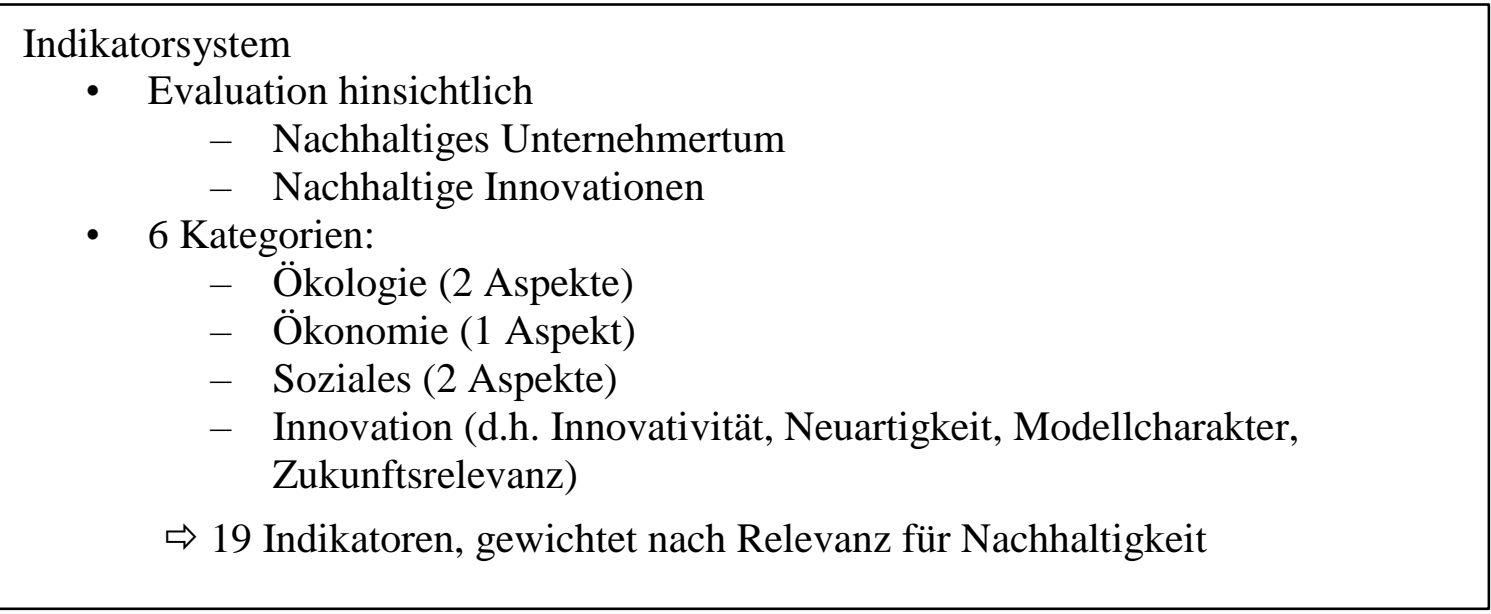

Abbildung 5: Aufbau des Evaluationssystems für nachhaltiges Unternehmertum

Indikatoren, die einen besonders hohen Beitrag zur nachhaltigen Entwicklung leisten, wurden mit der Gewichtung drei versehen. Die Energieeinsparung, Reduzierung des Materialverbrauches, Wiederverwertbarkeit des Materials, Reduzierung der klimaschädlichen Stoffe und Reduzierung der umweltschädlichen Stoffe sind Indikatoren, die den Grad der Umweltleistungen eines Projekts widerspiegeln. Sie untermauern die nachhaltig orientierte Ausrichtung einer Innovation und die Integration ökologischer Aspekte als zentrale Geschäftsziele in einem Unternehmen. Daher wurde für diese Indikatoren die Gewichtung drei festgelegt. Die Produktlebensdauer und eingebrachte Ressourcen sind ebenso Indikatoren der ökologischen Dimension, tragen jedoch die Gewichtung zwei. Die nachhaltige 
Beschaffenheit und der Nutzen für die Gesellschaft eines Produkts bzw. eines Prozesses sollen stärker gewichtet werden als die Produktlebensdauer. Diese Argumentation ist maßgeblich für die mittlere Bewertung. Bei dem Indikator „eingebrachte Ressourcen“ handelt es sich um eine relativ schwer bestimmbare und ambivalente Maßeinheit. Beispielsweise verzichtet ein Unternehmen beim Betrieb eines Holzspalters zwar auf Hydrauliköl und setzt stattdessen Klar- oder Reinwasserhydraulik ein. Auch wenn durch diese Methode die Umwelt geschont und Energie unter Umständen eingespart wird, handelt es sich bei der Ressource "Wasser" um ein Gut, das in Zukunft immer knapper sein wird. Um aber dennoch die Verwendung von umweltfreundlichen Betriebsmitteln zu berücksichtigen, erhält der Indikator „Eingebrachte Ressourcen“ die Gewichtung zwei.

Die ökonomische Dimension ist Bestandteil des Nachhaltigkeitsdreiecks und ebenfalls eine wichtige Säule. Jedoch ist davon auszugehen, dass ökonomische Aspekte Hauptbestandteil einer Innovation sind und generell fokussiert werden. Eine nachhaltige Umweltinnovation zeichnet sich dadurch aus, dass neben den ökonomischen Aspekten auch ökologische und soziale Leistungen verfolgt werden. Daher erhält der Indikator „Finanzielle Rentabilität“ nur die Gewichtung eins. Die Steigerung der Wettbewerbsfähigkeit könnte wiederum positive Effekte auf die Situation der Mitarbeiter und das Arbeitsklima ausüben (soziale Dimension) und wird dementsprechend mit der Ziffer zwei gewichtet. Der Indikator „Win-Win-Resultate“ bezieht sich sowohl auf eine erhöhte Kosteneinsparung als auch auf die Verringerung der Umweltbelastung. Aufgrund der äquivalenten Berücksichtigung der ökologischen Dimension wird für diesen Indikator gleichfalls die Gewichtung zwei festgelegt.

Die Befriedigung sozialer Bedürfnisse und der deutliche Nutzen für die Gesellschaft sind Bestandteil der Definition von „Nachhaltigkeit“. Unter diesem Gesichtspunkt werden die Indikatoren unter der vierten Kategorie, der sozialen Dimension, mit dem Faktor drei gewichtet. Gleichermaßen fallen die Indikatoren „Sicherung von bestehenden Arbeitsplätzen“ und „Schaffung von neuen Arbeitsplätzen“ unter die soziale Dimension. Dennoch wird für die beiden Indikatoren eine mittlere Gewichtung bestimmt. Grund hierfür ist, dass diese vergleichsweise eine geringe Anzahl an Personen betreffen, da beispielsweise selbst die Sicherung von mehreren tausend Arbeitsplätzen nur einen Bruchteil der gesamten Gesellschaft ausmacht.

Unter Berücksichtigung der Kriterien Effizienz und Innovativität wurden die Indikatoren „Modellcharakter“ und „subjektive Bewertung für die Zukunft“ mit der Gewichtung drei definiert. Demgemäß müsste der Indikator „Innovationsgrad des Produktes bzw. Prozesses“ die äquivalente Gewichtung erhalten. Aus der Nachhaltigkeitsperspektive betrachtet sollte die Neuartigkeit eines Prozesses oder Produktes nicht überbewertet werden, denn auch eine bereits geringfügige Veränderung kann enorme Effekte hervorrufen. Resultat ist nur eine mittlere Gewichtung dieses Indikators. Der Projektstand ist demzufolge wichtig, um zu beurteilen, inwiefern eine Idee Potential hat, tatsächlich realisiert zu werden. Wurde bereits ein Prototyp erstellt, ist diese Frage durchaus einfacher zu beantworten. Aus diesem Grund wurde für den Indikator „Projektstand“ die Gewichtung zwei bestimmt. Zuletzt erhält der Indikator „Verbesserungen in der Zukunft möglich“ die Gewichtung eins. Dieser Aspekt ist für den Erfolg und die Marktfähigkeit einer Prozess- oder Produktveränderung 
ausschlaggebend, jedoch ist die Bewertung oftmals eher subjektiv, da man zukünftige Entwicklungen nur bedingt vorhersehen kann.

Validiert wurde das Indikatorensystem durch Anwendung auf drei Praxisfälle, die einer Bewertung auf Basis des Indikatorensystems unterzogen wurden. Diese wurden ausgewählt, da Kofink (2010) sie bereits auf Basis der in Abb. 1 dargestellten Typologie klassifiziert hatte. Diese Information sowie ergänzende Recherchen waren die Basis einer Bewertung anhand des Indikatorensystems, wie sie folgend erläutert wird:

Das Unternehmen Starfort bietet Produkte wie Holzspalter, Hochdruckreiniger, Glastiegelverschlüsse und Baumstammsofas an. Die Besonderheit an dem angebotenen Sortiment ist der ökologische Maschinenbau, der hinter der Herstellung dieser Produkte steckt. Starfort ersetzt ölhydraulische Zylinder durch Klarwasserhydraulik. Durch diese Maßnahme möchte das Unternehmen Umweltstandards einhalten und konnte überdies eine Leistungssteigerung im Maschinenbau erreichen. Eine weitere Besonderheit von Starfort ist der vollständige Verzicht auf grelle Farbtöne aus Farbstoffen. Stattdessen werden vollbiologische Anstriche zur Lackierung der Geräte verwendet. Angesichts der Nischenmarktorientierung und Integration der Nachhaltigkeitsaspekte in den Kern der Geschäftstätigkeit ist das Unternehmen als Bioneer einzustufen.

Das Unternehmen Ecorecycling Felderer steht für die Verwendung von umweltschonenden Filtermethoden und für die Entwicklung neuartiger Reinigungsmethoden für Prozess- und Beregnungswasser. Die Filtersysteme werden europaweit in kleinen und großen Obstmagazinen und Verarbeitungsbetrieben für Obst eingesetzt. Weitere Forschungsschwerpunkte und Einsatzgebiete von Ecorecycling Felderer sind die Wasseraufbereitung, Schwimmbad Elektrolyse und Bodenanalysen. Diese neuen Technologien beeinflussen den Anwendungsmarkt nachhaltig, weshalb das Unternehmen in die Kategorie des Sustainable Entrepreneur einzuordnen ist.

Im Fokus des Unternehmens Mila steht das Angebot einer ökologisch wertvollen und ökologisch orientierten Produktpalette (Milchprodukte). Die nachhaltig orientierte Unternehmensführung hat sich dabei die Einhaltung wichtiger Umweltanforderungen zum Ziel gesetzt: Reduktion der Phosphate in Reinigungsmitteln, Reduzierung der CO2-Emission beim Fuhrpark und Vermeidung von Wärmeverlusten durch eine bessere Isolierung der Warmwasserleitungen. Überdies unterstützt Mila den Erhalt der Natur in der Region, indem das Unternehmen von über 3.500 Bergbauern aus der Umgebung Milch bezieht. Die Fokussierung auf den Milchprodukte Markt und die Umsetzung klarer Umweltanforderungen zeichnen das Unternehmen als Sozio-ökologischen Manager als Variante des nachhaltigen Unternehmertums aus (Kofink 2010). 


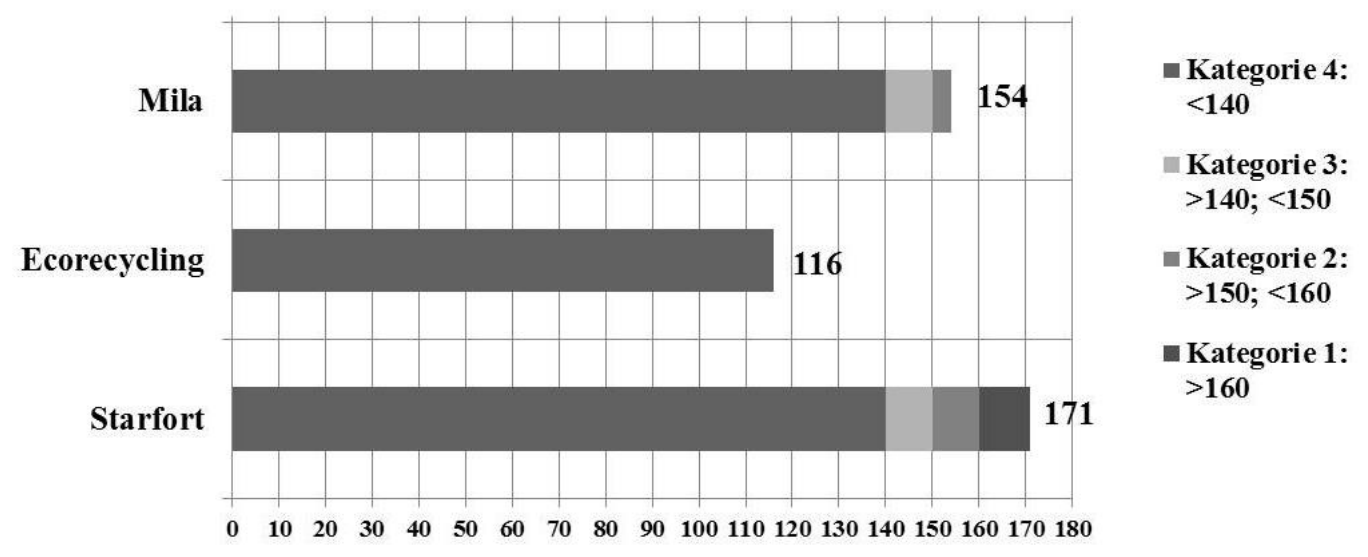

Abbildung 6: Auswertung der Praxisfälle anhand des Indikatorensystems

Die ermittelten Werte der drei Projekte durch das oben erläuterte Indikatorensystem variieren in einem Zahlenraum zwischen 116 und 171. Zur genaueren Analyse wurden die Ergebnisse in vier Kategorien unterteilt (s. Abbildung 6). Der vierten Kategorie $(<140)$ ist ein Projekt, der dritten Kategorie $(>140$; <150) kein Projekt und der zweiten Kategorie $(>150 ;<160)$ ein Projekt zuzuordnen. Das Projekt Starfort mit der höchsten Bewertung von 171 Punkten weißt eine hohe Wertung in den Kategorien „Innovationsgrad des Produktes bzw. des Prozesses“ sowie Neuartigkeitscharakter im Bereich der Nachhaltigkeitsinnovationen auf, wobei es sich hier konkret um eine technische Prozessveränderung handelt. Ecorecycling als „sustainable entrepreneur" erhält in dieser Auswertung die schlechteste Punktzahl im Indikatorsystem. Gründe hierfür können darin liegen, dass die Einstufungen von Kofink nicht im Detail erläutert werden, so dass zum einen ein Einstufungsfehler vorliegen könnte. Zum anderen besteht in der Matrix von Schaltegger/Petersen (2001) ein impliziter Tradeoff dahingehend, dass eine höhere Marktdurchdringung zwangsläufig eine „Verwässerung“ der Nachhaltigkeitsziele impliziert.

Ein weiteres Evaluationssystem zur Beurteilung nachhaltig orientierter Unternehmen ist die Sustainable Entrepreneurship Matrix (Schaltegger/Wagner 2008; Wagner/Schaltegger 2010; Schaltegger/Wagner 2011). Diese Methode eignet sich eher zur Untersuchung bereits etablierter Unternehmen und ist damit auch im vorliegenden Fall anwendbar.

Zur validierenden Analyse der Praxisfälle werden die Dimensionen/Indikatoren „Kategorien des Unternehmertums“ und „Nachhaltigkeitseinfluss“ der Sustainable Entrepreneurship Matrix detaillierter untersucht. Entsprechend des Bewertungskonzeptes zu „Kategorien des Unternehmertums“ können dabei (sozial-ökologische) Nachhaltigkeitsmanager, Bioneer und Sustainable Entrepreneur voneinander abgegrenzt werden. Anhand von Abbildung 7 lässt sich ablesen, dass keine völlige Korrelation zu Abbildung 6 besteht. Dies erfordert weitere Untersuchungen. Da die Gründe der Einstufung von Kofink (2010) nicht im Detail erläutert werden, ist nicht prüfbar, inwiefern dies einen Einfluss haben könnte. Denkbar wäre andererseits auch, dass in der Matrix von Schaltegger und Petersen (2001) ein impliziter Tradeoff dahingehend besteht, dass eine höhere Marktdurchdringung zwangsläufig eine „Verwässerung“ der Nachhaltigkeitsziele impliziert (vgl. auch die frühere Anmerkung zur 
„Light“-Ökologisierung). Auch dies würde die aus dem Indikatorensystem resultierenden Bewertungen zumindest teilweise erklären.

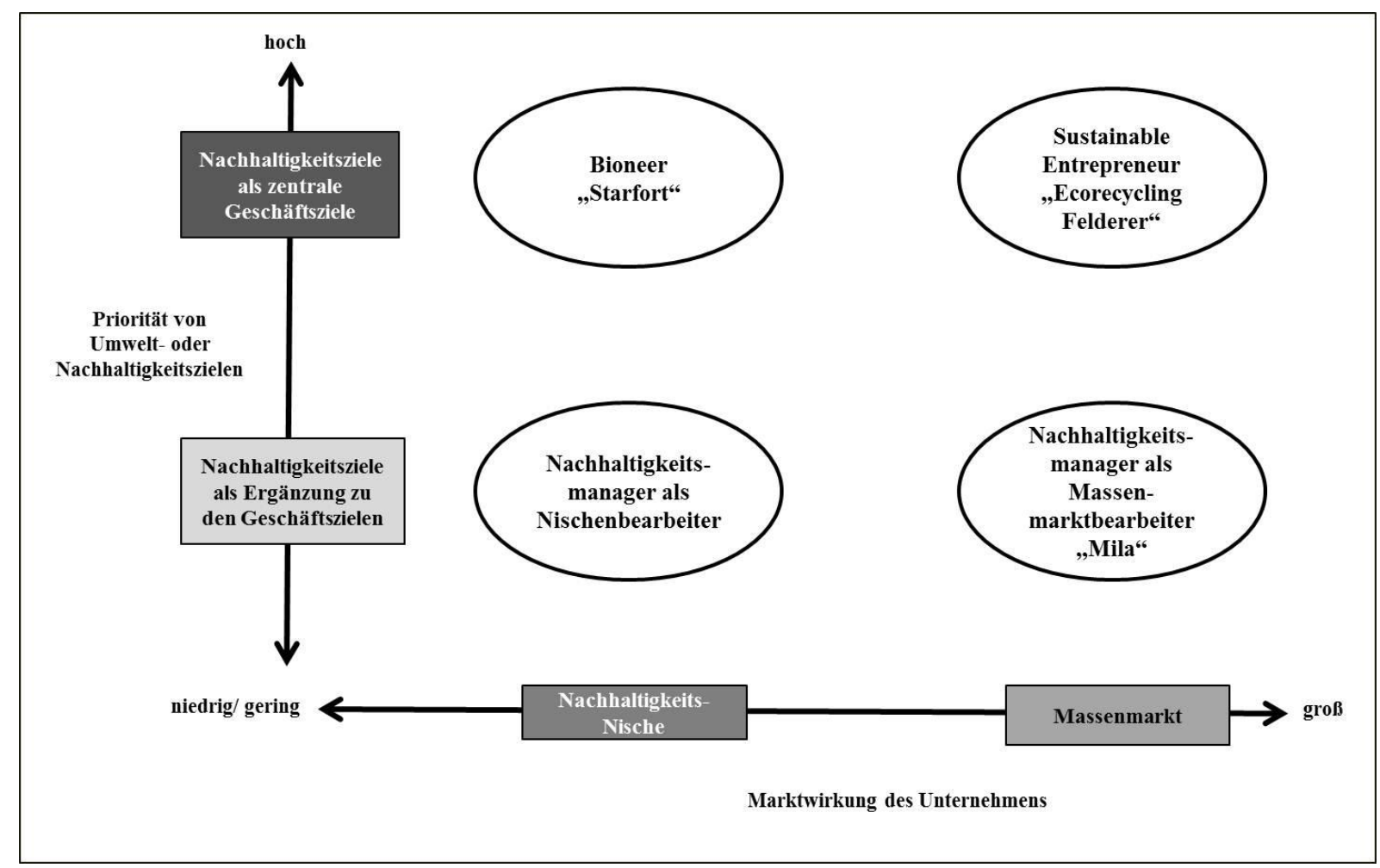

Abbildung 7: Typen nachhaltigen Unternehmertums (eigene, erweiterte Darstellung aufbauend auf Schaltegger/Petersen 2001) 


\section{Schlussfolgerungen}

Ausgehend von konzeptionellen und theoretischen Vorüberlegungen versucht das vorliegende Kapitel eine Konkretisierung der Begriffe des nachhaltigen Unternehmertums und der nachhaltigkeitsorientierten Innovation. Dabei wird einerseits auf bestehende Modelle zurückgegriffen, die dem Bedarf entsprechend erweitert und angepasst werden. Andererseits wird ein neues Indikatorensystem entwickelt, welches beide Bereiche integriert und ganzheitlich bewertet. Das Indikatorensystem wird an drei Fallstudien erprobt und dabei mit den aus bestehenden Konzepten abgeleiteten Kategorisierungen verglichen. Daraus ergibt sich, dass das entwickelte Indikatorensystem eine umfassende und valide Bewertung nachhaltigen Unternehmertums und nachhaltigkeitsorientierter Innovationen ermöglicht. Somit kann es als eine verlässliche Basis für zukünftige und umfangreichere Evaluationen in diesen Bereichen angesehen und angewendet werden. 


\section{Literatur}

Chapple, K., Kroll, C., Lester, T.W., Montero, S. (2011), Innovation in the Green Economy: An Extension of the Regional Innovation System Model?, Economic Development Quarterly, 25(1), 5-25

EIO Eco Innovation Observatory (2010), Methodological Report. Brussels: Europe Innova

Hauschildt J. (2004), Innovationsmanagement. München: Vahlen.

Hauschildt, J., Salomo, S. (2005), Je innovativer, desto erfolgreicher?, Journal für Betriebswirtschaft, 55, 3-20.

Hehl, W. (2010), Die bayerische Clusterstrategie und die Rolle des Umwelt-Technologischen Gründerzentrums Augsburgs (UTG), Vortrag an der Julius-Maximilians-Universität, Würzburg, 17.November.

Karl, H., Möller, A. (2004), Kooperationen zur Entwicklung von Umweltinnovationen Marktendogene Kooperationsdynamik und wirtschaftspolitische Kooperationsförderung, in: Horbach, J./Huber, J./Schulz, T. (Hrsg.): Nachhaltigkeit und Innovation, München: ökom, 191-218.

Kofink, L. (2010), Nachhaltigkeit und Unternehmertum, Fallstudien aus Südtirol, Institut für Regionalentwicklung und Standortmanagement, Vortrag auf dem Kompetenzforum Regionalmanagement 2010, Ingolstadt, 21. Oktober.

Konrad, W.; Nill, J. (2001), Innovationen für Nachhaltigkeit, Ein interdisziplinärer Beitrag zur konzeptionellen Klärung aus wirtschafts- und sozialwissenschaftlicher Perspektive. Berlin: Institut für ökologische Wirtschaftsforschung.

Marshall, A. (1920), Principles of economics. London: Macmillan

Porter, M. E. (1990), The competitive advantage of nations. New York: Free Press.

Porter, M.E., Delgado, M., Stern, S. (2010), Clusters and Entrepreneurship. Boston: Harvard Business School.

Porter, M.E. (2003), The economic performance of regions. Regional Studies, 37, 549-578.

Porter, M. E.; van der Linde, C. (1995), Toward a New Conception of the EnvironmentCompetitiveness Relationship; Journal of Economic Perspectives 9(4), 97-118

Reid A., Miedzinski M. (2008), Sectoral Innovation Watch in Europe - Eco-Innovation. Brussels: Europe Innova.

Rennings, K. (1998), Towards a Theory and Policy of Eco-Innovation-Neoclassic and (Co-) Evolutionary Perspectives, Center for European Economic Research (ZEW) Discussion Paper 98-24. 
Rennings, K. (2000), Redefining innovation - eco-innovation research and the contribution from ecological economics, Ecological Economics, 32, 319-332.

Schaltegger, S., Wagner, M. (2010), Sustainable Entrepreneurship and Sustainability Innovation: Categories and Interactions, Business Strategy and the Environment, 20, 222237.

Schaltegger, S.; Petersen, H. (2001), Ecopreneurship - Konzept und Typologie. Lüneburg/ Luzern: Center for Sustainability Management (CSM)/ Rio-Managementforum.

Schaltegger, S.; Wagner, M. (2011), Sustainable entrepreneurship and sustainability innovation: categories and interactions, Business Strategy and the Environment. 20(4), 222237

Schaltegger, S.; Wagner, M. (2008), Types of Sustainable Entrepreneurship and Conditions for Sustainability Innovation: From the administration of a technical challenge to the management of an entrepreneurial opportunity, in: Sharma, S/Starik, M./Wüstenhagen, R./Hamschmidt, J. (Hrsg.), Advances on Research in Corporate Sustainability, Boston: Edward Elgar, 27-48.

o.V. (2011), Umwelt-Technologie und Energie in Bayern, München: media mind GmbH.

Wagner, M. (2009a), Eco-Entrepreneurship - An Empirical Perspective based on Survey Data, Advances in the Study of Entrepreneurship, Innovation and Economic Growth, 20, 127 152.

Wagner, M. (2009b), Erfolgsfaktoren für Nachhaltigkeitsinnovationen: Qualitative und quantitative Befunde, Zeitschrift für Umweltrecht und Umweltpolitik, 2, 179-198.

Wagner, M.; Schaltegger, S. (2010), Classifying Entrepreneurship for the Public Good, Journal of Small Business \& Entrepreneurship, 23(3), 431-443

Wagner, M. (2012) The Role and Effectiveness of Environmental and Social Regulations in Creating Innovation Offsets and Enhancing Firm Competitiveness, in: Costantini, V./Mazzanti, M. (Hrsg.) The Dynamics of Environmental and Economic Systems, Berlin: Springer, Chapter 5.

Walley, E.E.; Taylor, D.W. (2002), Opportunists, Champions, Mavericks...? A Typology of Green Entrepreneurs; Greener Management International, 38, 31-43 